\title{
Face Authentication Using Adapted Local Binary Pattern Histograms
}

\author{
Yann Rodriguez and Sébastien Marcel \\ IDIAP Research Institute, Rue du Simplon 4, 1920 Martigny, Switzerland \\ \{rodrig, marcel\}@idiap.ch
}

\begin{abstract}
In this paper, we propose a novel generative approach for face authentication, based on a Local Binary Pattern (LBP) description of the face. A generic face model is considered as a collection of LBP-histograms. Then, a client-specific model is obtained by an adaptation technique from this generic model under a probabilistic framework. We compare the proposed approach to standard state-of-the-art face authentication methods on two benchmark databases, namely XM2VTS and BANCA, associated to their experimental protocol. We also compare our approach to two state-of-the-art LBP-based face recognition techniques, that we have adapted to the verification task.
\end{abstract}

\section{Introduction}

A face authentication (or verification) system involves confirming or denying the identity claimed by a person (one-to-one matching). In contrast, a face identification (or recognition) system attempts to establish the identity of a given person out of a closed pool of $N$ people (one-to- $N$ matching). Both modes are generally grouped under the generic face recognition term.

Authentication and identification share the same preprocessing and feature extraction steps and a large part of the classifier design. However, both modes target distinct applications. In authentication mode, people are supposed to cooperate with the system (the claimant wants to be accepted). The main applications are access control systems, such as computer or mobile devices log-in, building gate control, digital multimedia access. On the other hand, in identification mode, people are generally not concerned by the system and often even do not want to be identified. Potential applications include video surveillance (public places, restricted areas) and information retrieval (police databases, video or photo album annotation/identification).

Face recognition has been widely studied and is performing well in controlled lighting environment and on frontal faces. In real-world applications (unconstrained environment and non-frontal faces), face recognition does not yet achieve efficient results. Beside the pose of the subject, a major difficulty comes from the appearance variability of a given identity due to facial expressions, lighting, facial features (mustaches, glasses, make-up or other artefacts) or even the hair cut and skin color. The challenge of face recognition is then to extract relevant facial features which best discriminate individuals, in spite of the possible variations cited above.

A. Leonardis, H. Bischof, and A. Pinz (Eds.): ECCV 2006, Part IV, LNCS 3954, pp. 321-332 2006.

(C) Springer-Verlag Berlin Heidelberg 2006 
The problem of face authentication has been addressed by different researchers using various approaches. Thus, the performance of face authentication systems has steadily improved over the last few years. For a comparison of different approaches see [1]. These approaches can be divided mainly into discriminative approaches and generative approaches.

A discriminative approach takes a binary decision (whether or not the input face is a client) and considers the whole input for this purpose. Such holistic approaches are using the original gray-scale face image or its projection onto a Principal Component subspace (referred to as PCA or Eigenfaces [2]) or Linear Discriminant subspace (referred to as LDA or Fisherfaces [3]), or illuminationinvariant features [4,5] as input of a discriminative classifier such as Multi-Layer Perceptrons (MLPs) [6], Support Vector Machines (SVMs) 7] or simply a metric [8, 9]. Recently, it has been shown that generative approaches such as Gaussian Mixture Models (GMMs) [10] and Hidden Markov Models (HMMs) [11,12] were more robust to automatic face localization than the above discriminative methods. A generative approach computes the likelihood of an observation (a holistic representation of the face image) or a set of observations (local observations of particular facial features) given a client model and compares it to the corresponding likelihood given an impostor model. Finally, the decision to accept or reject a claim depends on a score (distance measure, MLP output or Likelihood ratio) which could be either above (accept) or under (reject) a given threshold.

In this paper, we propose a novel generative approach for face authentication, based on a Local Binary Pattern (LBP) description of the face. A generic face model is considered as a collection of LBP-histograms. Then, a client-specific model is obtained by an adaptation technique from this generic model under a probabilistic framework.

In the next section, we introduce the reader to the Local Binary Pattern (LBP) operator and its use to represent a face. Then, we describe the proposed approach. Finally, we provide experimental results comparing the proposed approach to state-of-the-art face verification techniques as well as to state-of-the-art LBP-based face identification techniques, on two databases, namely XM2VTS and BANCA, associated to their experimental protocol.

\section{Local Binary Patterns}

\subsection{The Local Binary Pattern Operator}

The local binary pattern (LBP) operator is a non-parametric $3 \times 3$ kernel which summarizes the local spacial structure of an image. It was first introduced by Ojala et al. 13] who showed the high discriminative power of this operator for texture classification. At a given pixel position $\left(x_{c}, y_{c}\right)$, LBP is defined as an ordered set of binary comparisons of pixel intensities between the center pixel and its eight surrounding pixels. The decimal form of the resulting 8-bit word (LBP code) can be expressed as follows (Figure 1):

$$
\operatorname{LBP}\left(x_{c}, y_{c}\right)=\sum_{n=0}^{7} s\left(i_{n}-i_{c}\right) 2^{n}
$$




\begin{tabular}{|l|l|l|}
\hline 83 & 75 & 126 \\
\hline 99 & 95 & 141 \\
\hline 91 & 91 & 100 \\
\hline
\end{tabular}
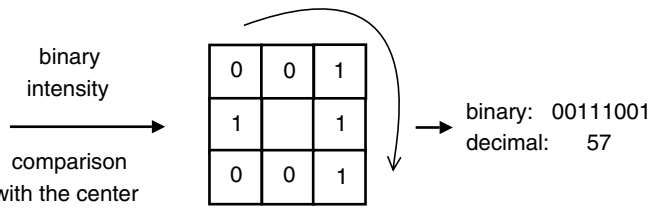

Fig. 1. The LBP operator

where $i_{c}$ corresponds to the grey value of the center pixel $\left(x_{c}, y_{c}\right), i_{n}$ to the grey values of the 8 surrounding pixels, and function $s(x)$ is defined as:

$$
s(x)=\left\{\begin{array}{lll}
1 & \text { if } & x \geq 0 \\
0 & \text { if } & x<0
\end{array}\right.
$$

Note that each bit of the LBP code has the same significance level and that two successive bit values may have a totally different meaning. Actually, The LBP code may be interpreted as a kernel structure index. By definition, the LBP operator is unaffected by any monotonic gray-scale transformation which preserves the pixel intensity order in a local neighbourhood.

Later, Ojala et al. 14] extended their original LBP operator to a circular neighbourhood of different radius size. Their $L B P_{P, R}$ notation refers to $P$ equally spaced pixels on a circle of radius $R$. In [14, they also noticed that most of the texture information was contained in a small subset of LBP patterns. These patterns, called uniform patterns, contain at most two bitwise 0 to 1 or 1 to 0 transitions (circular binary code). 11111111, 00000110 or 10000111 are for instance uniform patterns. They mainly represent primitive micro-features such as lines, edges, corners. $L B P_{P, R}^{u 2}$ denotes the extended LBP operator ( $u 2$ for only uniform patterns, labelling all remaining patterns with a single label).

Recently, new variants of LBP have appeared. For instance, Jin et al. 15 remarked that LBP features miss the local structure under certain circumstance, and thus they introduced the Improved Local Binary Pattern (ILBP). Huang et al. 16] pointed out that $\mathrm{LBP}$ can only reflect the first derivative information of images, but could not present the velocity of local variation. To solve this problem, they propose an Extended version of Local Binary Patterns (ELBP).

Due to its texture discriminative property and its very low computational cost, LBP is becoming very popular in pattern recognition. Recently, LBP has been applied for instance to face detection [15], face recognition [5, 4, image retrieval [17] or motion detection [18]. We finally point out that, approximately in the same time the original LBP operator was introduced by Ojala [13], Zabih and Woodfill [19] proposed a very similar local structure feature. This feature, called Census Transform, also maps the local neighbourhood surrounding a pixel. With respect to LBP, the Census Transform only differs by the order of the bit string. Later, the Census Transform has been extended to become the Modified Census Transform (MCT) 20]. Again, one can point out the same similarity between ILBP and MCT (also published at the same time).

\footnotetext{
${ }^{1}$ A more exhaustive list of applications can be found on Oulu University web site at: http://www.ee.oulu.fi/research/imag/texture/lbp/lbp.php
} 


\subsection{Face Representation with Local Binary Patterns}

In [4, Ahonen proposed a face recognition system based on a LBP representation of the face. The individual sample image is divided into $R$ small nonoverlapping blocks (or regions) of same size. Histograms of LBP codes $H^{r}$, with $r \in\{1,2, \ldots, R\}$ are calculated over each block and then concatened into a single histogram representing the face image. A block histogram can be defined as:

$$
H^{r}(i)=\sum_{x, y \in b l o c k_{r}} I(f(x, y)=i), i=1, \ldots, N,
$$

where $N$ is the number of bins (number of different labels produced by the LBP operator), $f(x, y)$ the LBP label 2 at pixel $(x, y)$ and $I$ the indicator function.

This model contains information on three different levels (Figure 2): LBP code labels for the local histograms (pixel level), local histograms (region level) and a concatened histogram which builds a global description of the face image (image level). Because some regions are supposed to contain more information (such as eyes), Ahonen propose an empirical method to assign weights to each region. For classification, a nearest-neighbour classifier is used with Chi square $\left(\chi^{2}\right)$ dissimilarity measure (see [4]).

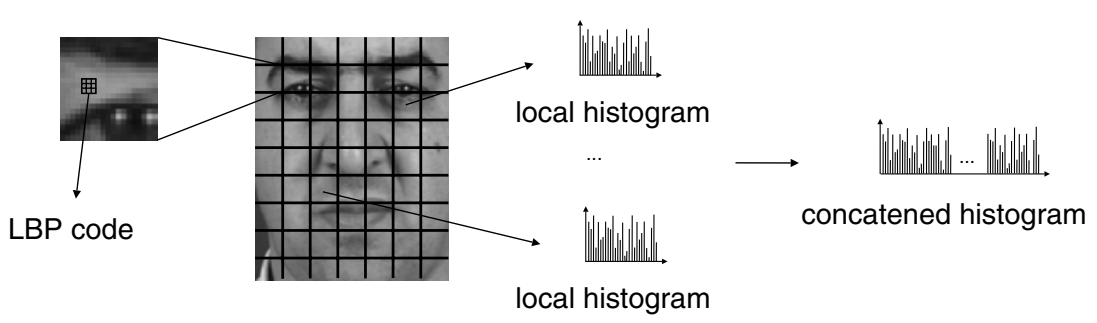

Fig. 2. LBP face description with three levels of information: pixel level (LBP code), region level (local histogram), image level (concatened histogram)

Following the work of Ahonen, Zhang et al. [5] underlined some limitations. First, the size and position of each region are fixed which limits the size of the available feature space. Second, the weighting region method is not optimal. To overcome these limitations, they propose to shift and scale a scanning window over pairs of images, extract the local LBP histograms and compute a dissimilarity measure between the corresponding local histograms. If both images are from the same identity, the dissimilarity measure are labelled as positive features, otherwise as negative features. Classification is performed with AdaBoost learning, which solves the feature selection and classifier design problem. Optimal position/size, weight and selection of the regions are then chosen by the boosting procedure. Comparative study with Ahonen's method showed similar results. Zhang et al.'s system uses however much less features (local LBP histograms).

${ }^{2}$ Note that $L B P(x, y)$, the LBP operator value, may not be equal to $f(x, y)$ which is the label assigned to the LBP operator value. With the $L B P_{P, R}^{u 2}$ operator, for instance, all non-uniform patterns are labelled with a single label. 


\section{Proposed Approach}

\subsection{Model Description}

In this paper, we propose a new generative model for face authentication, based on a LBP description of the face. Sample images are divided in $R$ non-overlapping block regions of same size. This block by block basis is mainly motivated by the success of some recent works [21,22, 12. Similar to [4, a histogram of LBP codes is computed for each block. However, this histogram is not seen as a static observation. We instead consider it as a probability distribution. Each block histogram is thus normalized: $\sum_{i} H^{r}(i)=1$, where $r \in\{1,2, \ldots, R\}$.

Given a claim for client $C$, let us denote a set of independent features $X=$ $\left\{x_{r}\right\}_{r=1}^{R}$, extracted from the given face image. If $\theta_{C}$ is the set of parameters to be estimated from sample $X$, we can define the likelihood of the claim coming from the true claimant $C$ as:

$$
\begin{aligned}
P\left(X \mid \theta_{C}\right) & =\prod_{r=1}^{R} p\left(x_{r} \mid \theta_{C}\right) \\
& =\prod_{r=1}^{R} p\left(x_{r} \mid \theta_{C_{1}}, \ldots, \theta_{C_{R}}\right) \\
& =\prod_{r=1}^{R} p\left(x_{r} \mid \theta_{C_{r}}\right),
\end{aligned}
$$

assuming that each block is independent and that $\theta_{C}$ can be decomposed as a set of independent parameters per block $\left(\theta_{C_{1}}, \ldots, \theta_{C_{R}}\right)$.

The next important step consists in choosing the function to estimate the likelihood functions $p\left(x_{r} \mid \theta_{C_{r}}\right)$. We chose a very simple and computationally inexpensive non parametric model: histogram of LBP codes. $x_{r}=\left\{l_{k}\right\}_{k=1}^{K}$ is thus defined as a set of $K$ labelled LBP code observations, where $K$ is the maximum number of kernels which can be computed in the block by the LBP operator. This value is constant because all blocks have the same size. Assuming that each LBP code observation is independent, we can thus develop further:

$$
\begin{aligned}
P\left(X \mid \theta_{C}\right) & =\prod_{r=1}^{R} p\left(x_{r} \mid \theta_{C_{r}}\right) \\
& =\prod_{r=1}^{R} p\left(l_{1}, \ldots, l_{K} \mid \theta_{C_{r}}\right) \\
& =\prod_{r=1}^{R} \prod_{k=1}^{K} p\left(l_{k} \mid \theta_{C_{r}}\right)
\end{aligned}
$$

where $p\left(l_{k} \mid \theta_{C_{r}}\right)=H_{C}^{r}\left(l_{k}\right)$, then:

$$
P\left(X \mid \theta_{C}\right)=\prod_{r=1}^{R} \prod_{k=1}^{K} H_{C}^{r}\left(l_{k}\right)
$$




\subsection{Client Model Adaptation}

In face verification, the available image gallery set of a given client is usually very limited (one to five images). To overcome this lack of training data, adaptation methods have been proposed, first for speaker verification [23] and then adapted for face verification [22,12. They consist in starting from a generic model and then adapting it to a specific client. This generic model, referred to as world model or universal background model, is trained with a large amount of data, generally independent of the client set, but as representative as possible of the client population to model. The most used technique of incorporating prior knowledge in the learning process is know as Maximum A Posteriori (MAP) adaptation [24]. MAP assumes that the parameters $\theta_{C}$ of the distribution $P\left(X \mid \theta_{C}\right)$ is a random variable which has a prior distribution $P\left(\theta_{C}\right)$. The MAP principle states that one should select $\hat{\theta}_{C}$ such that it maximizes its posterior probability density, that is:

$$
\begin{aligned}
\hat{\theta}_{C} & =\arg \max _{\theta_{C}} P\left(\theta_{C} \mid X\right) \\
& =\arg \max _{\theta_{C}} P\left(X \mid \theta_{C}\right) \cdot P\left(\theta_{C}\right) .
\end{aligned}
$$

Moreover, one can simplify further without loss of performance by using a global parameter to tune the relative importance of the prior. The parameter updating can be described from the general MAP estimation equations using constraints on the prior distribution presented in 24]:

$$
\hat{H}_{C}^{r}\left(l_{k}\right)=\alpha H_{W}^{r}\left(l_{k}\right)+(1-\alpha) H_{C}^{r}\left(l_{k}\right)
$$

where $H_{W}^{r}\left(l_{k}\right)$ is the feature value (bin $l_{k}$ of the histogram of block $r$ ) of the world model (prior), $H_{C}^{r}\left(l_{k}\right)$ is the current estimation (client training data) and $\hat{H}_{C}^{r}\left(l_{k}\right)$ is the updated feature value. The weighting factor $\alpha$ is chosen by crossvalidation. The client model is thus a combination of parameters estimated from an independent world model and from training samples. After adaptation, each block histogram $\hat{H}_{C}^{r}$ is normalized to remain a probability distribution.

\subsection{Face Verification Task}

Let us denote $\theta_{C}$ the parameter set for client model $C, \theta_{W}$ the parameter set for the world model and a set of feature $X$. The binary process of face verification can be expressed as follows:

$$
\Lambda(X)=\log P\left(X \mid \theta_{C}\right)-\log P\left(X \mid \theta_{W}\right)
$$

where $P\left(X \mid \theta_{C}\right)$ is the likelihood of the claim coming from the true claimant and $P\left(X \mid \theta_{W}\right)$ is the likelihood of the claim coming from an impostor. Given a decision threshold $\tau$, the claim is accepted when $\Lambda(X) \geq \tau$ and rejected when $\Lambda(X)<\tau . P(X \mid \theta$.$) is computed using Eq.10.$

\section{Experiments}

There are two main face authentication benchmark databases, namely XM2VTS and BANCA, which we briefly describe in this section. We will also provide comparative experiments with Ahonen and Zhang systems introduced in Section 2 . 


\subsection{Databases and Protocol}

The XM2VTS database 25] contains synchronized video and speech data from 295 subjects, recorded during four sessions taken at one month intervals. The subjects were divided into a set of 200 training clients, 25 evaluation impostors and 70 test impostors. We performed the experiments following the Lausanne Protocol Configuration I.

The BANCA database [26] was designed to test multi-modal identity verification with various acquisition devices under several scenarios (controlled, degraded and adverse). In the experiments described here we used the face images from the English corpora, containing 52 subjects. Each subject participated in 12 recording sessions in different conditions and with different cameras. Each of these sessions contains two video recordings: one true client access and one impostor attack. Five frontal face images were extracted from each video recording.

Whereas XM2VTS database contains face images in well controlled conditions (uniform blue background), BANCA is a much more challenging database with face images recorded in uncontrolled environment (complex background, difficult lightning conditions). See Figure 3 for example images of each database. To assess verification performance, the Half Total Error Rate (HTER) is generally used:

$$
\operatorname{HTER}(\theta)=\frac{\operatorname{FAR}(\theta)+\operatorname{FRR}(\theta)}{2} .
$$

where FAR if the false acceptance rate, FRR the false rejection rate and $\theta$ the decision threshold. To correspond to a realistic situation, $\theta$ is chosen a priori on the validation set at Equal Error Rate (EER).
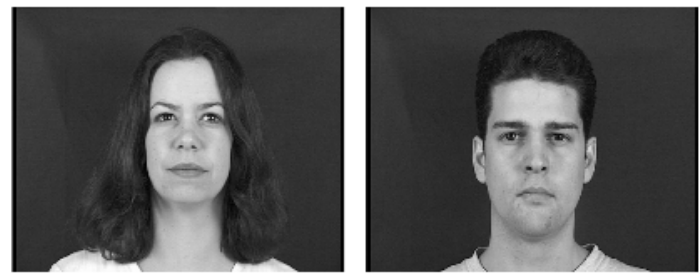

(a) XM2VTS (controlled conditions): uniform background and lighting
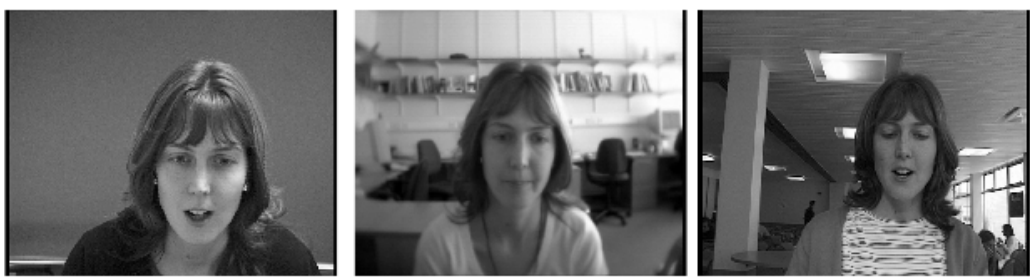

(b) BANCA English (uncontrolled conditions): complex background and lighting variability

Fig. 3. Comparison of XM2VTS (1) and BANCA (2) face image conditions 


\subsection{Experimental Setup}

For both XM2VTS and BANCA databases, face images are extracted to a size of $84 \times 68$ (rows $\times$ columns), according to the provided groundtruth eye positions. The cropped faces are then processed with the $L B P_{8,2}^{u 2}$ operator $(N=59$ labels $)$. The resulting $80 \times 64 L B P$ face images do not need any further lighting normalization, due to the gray-scale invariant property of LBP operators. In a block by block basis, the face images are decomposed in $8 \times 8$ blocks $(R=80$ blocks). Histograms of LBP codes are then computed over each block $r$ and normalized $\left(\sum_{i} H^{r}(i)=1\right.$, where $\left.i \in\{1,2, \ldots, N\}\right)$.

For experiments on XM2VTS database, we use all available training client images to build the generic model. For BANCA experiments, the generic model was trained with the additional set of images, referred to as world data (independent of the subjects in the client database). For both set of experiments, the adaptation factor $\alpha$ of Eq. 12 (client model adaptation) is selected on the respective validation sets.

For comparison purpose, we implemented the systems of Ahonen [4] and Zhang [5], briefly described in Section [2.2. Similarly, we used a $8 \times 8$ block decomposition and computed LBP histograms for each block with the $L B P_{8,2}^{u 2}$ operator.

\subsection{Results on XM2VTS Database}

Table1 1reports comparative results for Ahonen and Zhang systems, our proposed LBP/MAP histogram adaptation approach, as well as for two standard state-ofthe-art methods. LDA/NC 27] combines Linear Discriminant Analysis with Normalized Correlation (holistic representation of the face), while DCT/GMM [12] is a generative approach based on a modified version of the Discrete Cosine Transform and Gaussian Mixture Models (local description of the face).

We first remark that our method obtains state-of-the-art results. The main advantage of LBP/MAP is its very simple training procedure (only one parameter, the map factor). Training PCA and LDA matrices takes time (several hours) and is not trivial (initial dataset, data normalization, $\%$ of variance). Training GMM's is neither straightforward (choice of number of gaussians, iteration, floor factor, etc). We also note that compared to LDA/NC or DCTmod2/GMM, LBP/MAP does not need any lighting normalization preprocessing.

Table 1. HTER performance comparison (in \%) for two state-of-the-art methods (LDA/NC and DCT/GMM), Ahonen and Zhang systems and our proposed LBP/MAP histogram adaptation approach, on Configuration I of the XM2VTS database

\begin{tabular}{|c||c|}
\hline Models & Test set \\
\hline \hline LDA/NC [27] & 0.74 \\
DCTmod2/GMM [12] & 1.67 \\
\hline LBP Ahonen & 3.40 \\
LBP Zhang & 3.94 \\
\hline LBP/MAP & 1.42 \\
\hline
\end{tabular}


Compared to the two other LBP methods, LBP/MAP performs clearly better. However, it must be noted that these methods have been originally designed for face identification task. We finally point out that as reported in [5] for identification, Ahonen and Zhang methods give similar results.

\subsection{Results on BANCA Database}

Table 2 reports results from the same systems than those in Table1, but the LBP Zhang system. This is because Huang et al. 28] recently proposed an improved version of Zhang et al. system [5], based on a modified version of the boosting procedure called JSBoost, and provided results on BANCA. We then denote this method LBP/JSBoost. Unfortunately they only gave results with Protocol G.

Table 2. HTER performance comparison (in \%) for two state-of-the-art methods (LDA/NC and DCT/GMM), Ahonen and LBP/JSBoost systems and our proposed LBP/MAP histogram adaptation approach, for Protocol Mc, Ud, Ua, P and G of the BANCA database. Boldface indicates the best result for a protocol.

\begin{tabular}{|c||c|c|c|c|c|}
\hline \multicolumn{1}{|c||}{ Models } & \multicolumn{5}{c|}{ Protocols } \\
& $\mathrm{Mc}$ & $\mathrm{Ud}$ & $\mathrm{Ua}$ & $\mathrm{P}$ & $\mathrm{G}$ \\
\hline \hline LDA/NC [27 & $\mathbf{4 . 9}$ & 16.0 & 20.2 & $\mathbf{1 4 . 8}$ & 5.2 \\
DCTmod2/GMM 12 & 6.2 & 23.7 & $\mathbf{1 7 . 6}$ & 18.6 & - \\
\hline LBP Ahonen & 8.3 & 14.3 & 23.1 & 20.8 & 10.4 \\
LBP/JSBoost [28] & - & - & - & - & 10.7 \\
\hline LBP/MAP & 7.3 & $\mathbf{1 0 . 7}$ & 22.6 & 19.2 & $\mathbf{5 . 0}$ \\
\hline
\end{tabular}

Looking at the last three rows of Table 2, we notice again that our generative method performs better that the two other LBP-based methods for all conditions. On protocol $\mathrm{G}$, where more client training data is available, LBP/MAP clearly outperforms the improved version of Zhang system (LBP/JSBoost).

The LDA/NC model obtains the best result in matched condition (Mc). For uncontrolled environment, LBP/MAP shows the best results in degraded condition (Ud). This is certainly due to the illumination invariant property of LBP features. Indeed, in controlled (Mc) and adverse (Ua) conditions, the lighting is almost uniform on the faces, whereas in degraded condition, the left part of most of the faces are illuminated.

In adverse condition, the recording camera was below the horizontal plan of the head. Moreover, people were not really looking at the camera, leading to a distorsion effect. The local representation of the face in the DCTmod2/GMM model can probably explain why this approach outperforms the other holistic model 3 Finally, it is interesting to notice that no single model appears to be the best one in all conditions.

\footnotetext{
${ }^{3}$ Although based on local histograms, all three LBP methods are holistic because of
} the concatened histogram representing the face. 


\section{Conclusion}

In this paper, we proposed a novel generative approach for face authentication, based on a Local Binary Pattern (LBP) description of the face. A generic face model was considered as a collection of LBP-histograms. Then, a client-specific model was obtained by an adaptation technique from this generic model under a probabilistic framework. Experiments were performed on two databases, namely XM2VTS and BANCA, associated to their experimental protocol. Results have shown that the proposed approach performs better than stateof-the-art LBP-based face recognition techniques and is much faster than other state-of-the-art face verification techniques that perform similarly than the proposed approach.

Experimental results on BANCA database show that our method was performing well in uncontrolled lighting condition (Ud), due to the illumination invariance property of the LBP operator. However, our system was limited in the adverse condition (Ua), whereas the local approach (DCTmod2/GMM) was performing best. An interesting future work would be to investigate the use of LBP features with more appropriate Graphical Models, similar to the above GMM framework. This also motivated by the fact that local approaches have shown more robustness to non-perfect face localization than holistic approaches, which is particularly important for real-life automatic systems.

\section{Acknowledgment}

This research has been carried out in the framework of the Swiss NCCR project (IM)2. This publication only reflects the authors' views. All experiments were done using the Torch3 library [29] and the Torch3vision packag@4. The authors would like to thank Samy Bengio for corrections and useful suggestions.

\section{References}

1. Messer, K., Kittler, J., Sadeghi, M., Hamouz, M., Kostyn, A., Marcel, S., Bengio, S., Cardinaux, F., Sanderson, C., Poh, N., Rodriguez, Y., Kryszczuk, K., Czyz, J., Vandendorpe, L., Ng, J., Cheung, H., Tang, B.: Face authentication competition on the BANCA database. In: Proceedings of the International Conference on Biometric Authentication (ICBA), Hong Kong (2004)

2. Turk, M., Pentland, A.: Eigenface for recognition. Journal of Cognitive Neuroscience 3(1) (1991) 70-86

3. Belhumeur, P., Hespanha, J.P., Kriegman, D.J.: Eigenfaces vs. Fisherfaces: Recognition using class specific linear projection. In: ECCV'96. (1996) 45-58 Cambridge, United Kingdom.

4. Ahonen, T., Hadid, A., Pietikäinen, M.: Face recognition with local binary patterns. In: Proc. 8th European Conference on Computer Vision (ECCV), Prague, Czech Republic. (2004) 469-481

${ }^{4}$ See: http://www.idiap.ch/ marcel/en/torch3/introduction.php 
5. Zhang, G., Huang, X., Li, S., Wang, Y., Wu, X.: Boosting local binary pattern (LBP)-based face recognition. In: Proc. Advances in Biometric Person Authentication: 5th Chinese Conference on Biometric Recognition, SINOBIOMETRICS 2004Guangzhou, China. (2004) 179-186

6. Marcel, S.: A symmetric transformation for LDA-based face verification. In: Proceedings of the 6th International Conference on Automatic Face and Gesture Recognition, IEEE Computer Society Press (2004)

7. Jonsson, K., Matas, J., Kittler, J., Li, Y.: Learning support vectors for face verification and recognition. In: 4th International Conference on Automatic Face and Gesture Recognition. (2000) 208-213

8. Li, Y., Kittler, J., Matas, J.: On matching scores of LDA-based face verification. In Pridmore, T., Elliman, D., eds.: Proceedings of the British Machine Vision Conference BMVC2000, British Machine Vision Association (2000)

9. Kittler, J., Ghaderi, R., Windeatt, T., Matas, G.: Face verification via ECOC. In: British Machine Vision Conference (BMVC01). (2001) 593-602

10. Cardinaux, F., Sanderson, C., Marcel, S.: Comparison of MLP and GMM classifiers for face verification on XM2VTS. In: 4th International Conference on Audioand Video-Based Biometric Person Authentication (AVBPA), Guilford, UK (2003) 911-920

11. Nefian, A., Hayes, M.: Face recognition using an embedded HMM. In: Proceedings of the IEEE Conference on Audio and Video-based Biometric Person Authentication (AVBPA). (1999) 19-24

12. Cardinaux, F., Sanderson, C., Bengio, S.: Face verification using adapted generative models. In: IEEE Conference on Automatic Face and Gesture Recognition (AFGR). (2004)

13. Ojala, T., Pietikäinen, M., Harwood, D.: A comparative study of texture measures with classification based on feature distributions. Pattern Recognition 29 (1996)

14. Ojala, T., Pietikäinen, M., Mäenpää, T.: Multiresolution gray-scale and rotation invariant texture classification with local binary patterns. IEEE Transactions on Pattern Analysis and Machine intelligence 24 (2002) 971-987

15. Jin, H., Liu, Q., Lu, H., Tong, X.: Face detection using improved LBP under bayesian framework. In: Proc. Third International Conference on Image and Graphics (ICIG), Hong Kong, China. (2004) 306-309

16. Huang, X., Li, S., Wang, Y.: Shape localization based on statistical method using extended local binary pattern. In: Proc. Third International Conference on Image and Graphics (ICIG), Hong Kong, China. (2004) 184-187

17. Takala, V., Ahonen, T., Pietikäinen, M.: Block-based methods for image retrieval using local binary patterns. In: Proc. 14th Scandinavian Conference on Image Analysis (SCIA), Joensuu, Finland. (2005) 882-891

18. Heikkilä, M., Pietikäinen, M., Heikkilä, J.: A texture-based method for detecting moving objects. In: Proc. the 15th British Machine Vision Conference (BMVC), London, UK. Volume 1. (2004) 187-196

19. Zabih, R., Woodfill, J.: A non-parametric approach to visual correspondence. IEEE Transactions on Pattern Analysis and Machine intelligence (1996)

20. Fröba, B., Ernst, A.: Face detection with the modified census transform. In: IEEE Conference on Automatic Face and Gesture Recognition (AFGR). (2004)

21. Lucey, S., Chen, T.: A GMM parts based face representation for improved verification through relevance adaptation. In: Proc. IEEE Int. Conf. on Computer Vision and Pattern Recognition (CVPR), Washington D.C., USA. (2004)

22. Sanderson, C., Paliwal, K.: Fast features for face authentication under illumination direction changes. Pattern Recognition Letters (2003) 2409-2419 
23. Reynolds, D.A., Quatieri, T.F., Dunn, R.B.: Speaker verification using adapted gaussian mixture models. Digital Signal Processing 10(1-3) (2000)

24. Gauvain, J.L., Lee, C.H.: Maximum a posteriori estimation for multivariate gaussian mixture observation of markov chains. In: IEEE Transactions on Speech Audio Processing. Volume 2. (1994) 291-298

25. Messer, K., Matas, J., Kittler, J., Luettin, J., Maitre, G.: XM2VTSDB: The extended M2VTS database. In: Second International Conference on Audio and Video-based Biometric Person Authentication. (1999)

26. Bailly-Baillière, E., Bengio, S., Bimbot, F., Hamouz, M., Kittler, J., Mariéthoz, J., Matas, J., Messer, K., Popovici, V., Porée, F., Ruiz, B., Thiran, J.P.: The BANCA database and evaluation protocol. In: 4th International Conference on Audio- and Video-Based Biometric Person Authentication (AVBPA), Guilford, UK (2003)

27. Sadeghi, M., Kittler, J., Kostin, A., Messer, K.: A comparative study of automatic face verification algorithms on the banca database. In: 4th International Conference on Audio- and Video-Based Biometric Person Authentication (AVBPA), Guilford, UK (2003) 35-43

28. huang, X., Li, S.Z., Wang, Y.: Jensen-shannon boosting learning for object recognition. In: Proc. IEEE Int. Conf. on Computer Vision and Pattern Recognition (CVPR), San Diego, USA. (2005)

29. Collobert, R., Bengio, S., Mariéthoz, J.: Torch: a modular machine learning software library. Technical Report 02-46, IDIAP, Martigny, Switzerland (2002) 